OPEN ACCESS

Edited by:

Lorenzo Loffredo,

Sapienza University of Rome, Italy

Reviewed by:

Wei Li,

Marshall University, United States

Coen Maas,

University Medical Center Utrecht, Netherlands

*Correspondence:

Yurong Xing

xingyur@163.com

tThese authors have contributed equally to this work

Specialty section:

This article was submitted to Hematology,

a section of the journal

Frontiers in Medicine

Received: 17 March 2020 Accepted: 29 May 2020

Published: 21 July 2020

Citation:

Liu Y, Zhu Y, Jia W, Sun D, Zhao L, Zhang C, Wang C, Lyu Q, Chen Y,

Chen G, Bo Y and Xing Y (2020) Association of the Total White Blood Cell, Neutrophils, and Monocytes Count With the Presence, Severity, and Types of Carotid Atherosclerotic

Plaque. Front. Med. 7:313

doi: 10.3389/fmed.2020.00313

\section{Association of the Total White Blood Cell, Neutrophils, and Monocytes Count With the Presence, Severity, and Types of Carotid Atherosclerotic Plaque}

\author{
Yanhua Liu ${ }^{1 \dagger}$, Yongjian Zhu ${ }^{2 \dagger}$, Wenrui $\mathrm{Jia}^{3}$, Dan Sun ${ }^{3}$, Li Zhao ${ }^{3}$, Chen Zhang ${ }^{3}$, \\ Cuicui Wang ${ }^{3}$, Quanjun Lyu ${ }^{1}$, Yuming Chen ${ }^{4}$, Gaiyun Chen ${ }^{1}$, Yacong Bo ${ }^{1}$ and \\ Yurong Xing ${ }^{3 *}$
}

${ }^{1}$ Department of Nutrition, The First Affiliated Hospital of Zhengzhou University, Zhengzhou, China, ${ }^{2}$ Department of Cardiology, The First Affiliated Hospital of Zhengzhou University, Zhengzhou, China, ${ }^{3}$ Department of Physical Center, The First Affiliated Hospital of Zhengzhou University, Zhengzhou, China, ${ }^{4}$ Guangdong Provincial Key Laboratory of Food, Nutrition and Health, School of Public Health, Sun Yat-sen University, Guangzhou, China

Background: Previous studies have indicated that white blood cells (WBCs) might contribute to the development of atherosclerosis. However, the associations of WBCs and WBC subgroups with carotid atherosclerotic plaque (CAP) have not been compared.

Methods: A cross-sectional study including 3,569 healthy Chinese adults was conducted between January 2016 and December 2018 in Zhengzhou, China, to explore the associations of WBC and WBC subtypes with the presence, severity, and types of CAPs. Fasting peripheral venous blood was collected for measurement of the total WBC and WBC subtype counts. The size, composition, and types of CAPs in the common carotid artery, the internal carotid artery, and the external carotid artery were measured bilaterally using B-mode ultrasound.

Results: The total WBC, neutrophil, and monocyte counts showed significant associations with the presence of CAPs in men, but not in women, with the adjusted odds ratios $(95 \% \mathrm{Cl})$ in the highest (compared to the lowest) quartile 1.99 (1.33-2.97), $1.65(1.10-2.47)$, and $2.17(1.41-3.18)\left(P_{\text {trend }}=0.004, P_{\text {trend }}=0.004\right.$, and $P_{\text {trend }}$ $<0.001)$, respectively. The three leukocyte counts were also significantly associated with the severity of CAPs, as judged by the count of CAPs, maximal internal carotid plaque thickness, and the plaque score (all $P<0.01, P_{\text {trend }}<0.05$ ). Compared with individuals without CAPs, those with echolucent plaques had significantly increased total WBC and neutrophil counts, whereas those with polytype plaques had a significantly increased monocyte count.

Conclusion: WBC, neutrophil, and monocyte counts were significantly associated with the presence, severity, and types of CAPs in a healthy Chinese population.

\footnotetext{
Keywords: atherosclerosis, carotid atherosclerotic plaque, white blood cell count, neutrophils, monocytes
} 


\section{INTRODUCTION}

Atherosclerosis is a strong risk factor for cardiovascular and cerebrovascular diseases, which are the leading causes of death and disability worldwide (1), and have become an important public health concern in China (2). Although individuals with atherosclerosis are usually without symptoms in the early stages, atherosclerosis can progress at their young age and throughout the lifetime. Atherosclerosis involves the progressive narrowing of the vessel's lumens, which may lead to acute cardiac ischemia, myocardial infarction, or stroke. The carotid intima-media thickness (CIMT), measured by a carotid ultrasound, has been used as a surrogate indicator for atherosclerosis. However, CIMT provided less clear evidence for atherosclerotic infiltration in the vessel wall than a significant carotid atherosclerotic plaque (CAP) does (3). Several studies have demonstrated that, compared to the mean IMT, CAP could predict the occurrence of cardiovascular and cerebrovascular diseases more accurately.

Recently, it has been recognized that atherogenesis is not only a passive injury with infiltration of lipids but is also an active inflammatory process. The relationship of the white blood cell (WBC) count with inflammation in various diseases has been investigated (4-6). WBCs comprised the subtypes of neutrophils, lymphocytes, monocytes, eosinophils, and basophils, each of which plays a distinct inflammatory and immunologic role and may contribute differently to the development of atherosclerosis (7). Several studies have confirmed the association of the WBC count with the risk of cardiac cerebral disease $(8,9)$. Recruitment of leukocytes to the vascular endothelium wall represents an early stage in the initiation and development of atherosclerosis (10). Although increased leukocytes have been reported to correlate with CAP, the associations of the WBC subgroups with CAPs have not been compared (11). As the WBC count is an inexpensive and widely used test, we conducted the current study to more fully explore the associations between WBCs and the presence, severity, and types of CAPs.

\section{METHODS}

\section{Design and Study Population}

This cross-sectional study included 3,569 healthy Chinese subjects (2,464 men and 1,105 women) aged 18-97 years, and the associations of the total WBC and WBC subtypes with CAPs were investigated. All subjects were recruited through a general health screening programme between January 2016 and December 2018 in Zhengzhou, China. Individuals were excluded for the following reasons: (i) a history of cardiovascular disease, including coronary heart disease, stroke, thromboembolic disease, congestive heart failure, or peripheral arterial disease; (ii) cancer; (iii) infectious disease; (iv) serious liver or renal disorders; and (v) currently on medication. The current study was approved by the Research Ethical Committee of the First Affiliated Hospital of Zhengzhou University, and written informed consent was obtained from each participant at the time of enrollment.

\section{Parameter Measurements}

Weight and height were measured using an autoanthropometer, and body mass index (BMI) was calculated with the formula: weight in kilograms divided by height in meter squared. Blood pressure was measured by an electronic sphygmomanometer after the subjects had been sitting in a relaxed position for $10 \mathrm{~min}$. Data on smoking, drinking, and history of diseases were collected using a standard physician-administered questionnaire. Smokers and drinkers were defined as participants who smoked one or more cigarettes per day and drank alcohol once or more a week for at least 6 months, respectively.

Fasting peripheral vein blood $(5 \mathrm{ml})$ was drawn in EDTAanticoagulant tubes. After centrifugation at $1,500 \times \mathrm{g}$ for $15 \mathrm{~min}$ at $4^{\circ} \mathrm{C}$, plasma and blood cells were separated within $2 \mathrm{~h}$. The total WBC and WBC subtype (neutrophils, lymphocytes, monocytes, eosinophils, and basophils) counts and their percentages were assayed automatically by a blood cell counter (Beckman Coulter, Miami, FL, USA). Standard quality control procedures were followed in the laboratory. The plasma levels of total cholesterol (TC), triglycerides (TG), high-density lipoprotein cholesterol (HDLc), lowdensity lipoprotein cholesterol (LDLc), glucose, and uric acid were measured using a fully automatic analyzer (Roche Molecular Systems, Inc., Basel, Switzerland) module Cobas 8000 (C701/C702/C502). The coefficient of variation for repeated measurements on the blood samples was maintained at a level of $2.5 \%$.

\section{Atherosclerosis Assessment}

The presence of CAPs was measured bilaterally by B-mode ultrasound using an Aplio 300 ultrasound system (Toshiba Medical Systems, Tokyo, Japan). Measurements were taken in the far wall of the common carotid artery (CCA), the internal carotid artery (ICA), and the external carotid artery (ECA). When the subjects have lain in a supine position, the image of the extracranial carotid arteries were acquired in the longitudinal (anterior, lateral, and posterior views) and transverse planes. CAP was defined as a local protrusion of at least $0.5 \mathrm{~mm}$, a $50 \%$ local thickening of IMT, or a CIMT $>1.5 \mathrm{~mm}$ (12).

When a plaque was imaged, the view of the thickest part of the plaque was frozen and an electronic cursor was used to measure the intimal-medial wall thickness (including the plaque), recording it as the maximal internal carotid plaque thickness (MICPT) for that artery. When no plaque was identified, the MICPT was recorded as zero (13). The plaque score was thereafter calculated by summing the measurements of plaque thickness in both carotid arteries (14).

According to ultrasound imaging, plaques were classified into three types: echolucent (low grayscale median, lipid-rich), echogenic (high grayscale median, mostly occupied by calcified areas), or heterogeneous (mixed echolucent and echogenic) (15). A plaque was classified as a polytype when more than one composition was present. All CAP measurements were conducted by the same senior physician using B-mode ultrasound, and strict quality control procedures were followed during image acquisition. 


\section{Statistical Analyses}

The data are presented as means with standard deviations (SDs) for the continuous variables and count with frequencies for the categorical variables. Student's $t$-test and Pearson's chi-square test were conducted, as appropriate. Pearson's correlation analysis was used to assess the relationship between blood cell counts and lipid profiles.

The study participants were divided into quartiles according to their total and differential WBC counts and by gender. Binary logistical regression models were applied to estimate the association of total and differential WBC counts with the presence of CAPs. Three models were developed: model 1, adjusted for age and BMI; model 2, further adjusted for fasting plasma lipid profiles, fasting plasma glucose, systolic blood pressure (SBP), diastolic blood pressure (DBP), and uric acid; and model 3, further adjusted for drinker and smoker (only for men). The potential non-linear associations of the blood cell counts with the presence of CAPs were assessed by restricted cubic spline with three knots.

The study participants were also divided into quartiles according to their MICPT and plaque scores. The lowest two quartiles (Q1 and Q2) were combined into one category (Q1-Q2); the MICPT and plaque scores of all participants in these quartiles were recorded as zero. Analysis of covariance (ANCOVA) was adopted to compare the mean total and differential WBC counts according to the sum and the composition of CAPs and the quartiles of MICPT and plaque score. The same covariates were adjusted for the ANCOVA as those in the regression analysis. Pairwise comparisons were performed using the Bonferroni test.

The analyses were performed with SPSS 13.0 (SPSS, Inc., Chicago, USA) and R 3.6.0 (R Core Team, Vienna, Austria). Two-sided $P<0.05$ indicated statistical significance.

\section{RESULTS}

\section{Baseline Characteristics}

Of the 3,569 subjects, 331 were excluded in accordance with the exclusion criteria and due to incomplete data. Thus, a total of 3,238 participants were included in the analysis. Table 1 summarizes the baseline characteristics of the study participants by gender. The 2,249 men and 989 women had mean ages of $50.6 \pm 10.8$ and $51.2 \pm 10.8$ years, respectively. Total WBC and most of the differential WBC counts were higher in men than in women $(P \leq 0.010)$. The percentages of subjects with plaques in any carotid artery, CCA, ICA, or ECA were also higher in men than in women $(P<0.001)$ : 49.6, 46.6, 14.2, and $3.7 \%$ for men and $31.9,29.0,6.5$, and $1.4 \%$ for women, respectively. Sex differences were also found in the MICPT, plaque score, and the count and composition of CAPs $(P<$ 0.001). Pearson's analysis revealed that the total WBC and neutrophil counts were significantly associated with TG and HDL-c $(P<0.05)$, but not TC or LDL-c, and the monocyte count was only significantly associated with TG $(P<0.05$; Figure S1).
TABLE 1 | Characteristics of the study participants.

\begin{tabular}{|c|c|c|c|c|c|c|c|}
\hline & \multicolumn{3}{|c|}{ Men } & \multicolumn{3}{|c|}{ Women } & \multirow[t]{2}{*}{$\boldsymbol{P}$} \\
\hline & Mean & $S D$ & $N$ & Mean & $S D$ & $N$ & \\
\hline Age (years) & 50.6 & 10.8 & 2,249 & 51.2 & 10.8 & 989 & 0.154 \\
\hline Height (cm) & 172.5 & 5.7 & 1,948 & 160.7 & 5.9 & 824 & $<0.001$ \\
\hline Weight (kg) & 77.8 & 10.0 & 1,949 & 62.1 & 8.8 & 826 & $<0.001$ \\
\hline $\mathrm{BMI}\left(\mathrm{kg} / \mathrm{m}^{2}\right)$ & 26.1 & 2.9 & 1,948 & 24.0 & 3.2 & 824 & $<0.001$ \\
\hline $\mathrm{SBP}(\mathrm{mmHg})$ & 132.6 & 17.7 & 1,934 & 127.3 & 19.8 & 808 & $<0.001$ \\
\hline DBP (mmHg) & 82.2 & 12.9 & 1,933 & 74.4 & 12.8 & 808 & $<0.001$ \\
\hline Plasma glucose (mmol/L) & 5.40 & 1.62 & 2,147 & 5.16 & 1.15 & 925 & $<0.001$ \\
\hline Uric acid (mmol/L) & 348.9 & 91.5 & 2,197 & 253.4 & 75.2 & 975 & $<0.001$ \\
\hline TC (mmol/L) & 4.67 & 1.07 & 2,126 & 4.73 & 1.05 & 959 & 0.158 \\
\hline TG (mmol/L) & 2.05 & 4.57 & 2,146 & 1.42 & 0.96 & 961 & $<0.001$ \\
\hline HDLc (mmol/L) & 1.30 & 0.47 & 2,143 & 1.50 & 0.46 & 953 & $<0.001$ \\
\hline LDLc (mmol/L) & 2.95 & 0.87 & 2,101 & 2.99 & 0.81 & 931 & 0.212 \\
\hline Total WBC Count $\left(\times 10^{9} / \mathrm{L}\right)$ & 6.44 & 1.68 & 2,236 & 5.82 & 1.55 & 987 & $<0.001$ \\
\hline Neutrophils $\left(\times 10^{9} / \mathrm{L}\right)$ & 3.79 & 1.53 & 2,248 & 3.38 & 1.16 & 989 & $<0.001$ \\
\hline Neutrophils (\%) & 57.7 & 7.74 & 2,245 & 57.5 & 7.90 & 988 & 0.555 \\
\hline Lymphocytes (×109/L) & 2.04 & 0.58 & 2,247 & 1.90 & 0.57 & 986 & $<0.001$ \\
\hline Lymphocytes (\%) & 32.0 & 7.07 & 2,221 & 33.3 & 7.16 & 981 & $<0.001$ \\
\hline Monocytes (×109/L) & 0.46 & 0.24 & 2,243 & 0.38 & 0.25 & 988 & $<0.001$ \\
\hline Monocytes (\%) & 7.09 & 1.83 & 2,245 & 6.49 & 1.63 & 989 & $<0.001$ \\
\hline Eosinophils (×109/L) & 0.43 & 1.17 & 2,247 & 0.33 & 0.86 & 988 & 0.01 \\
\hline Eosinophils (\%) & 2.34 & 2.25 & 2,248 & 1.94 & 1.86 & 988 & $<0.001$ \\
\hline Basophils (×109/L) & 0.05 & 0.08 & 2,246 & 0.05 & 0.08 & 989 & 0.286 \\
\hline Basophils (\%) & 0.52 & 0.25 & 2,248 & 0.53 & 0.22 & 989 & 0.302 \\
\hline MICPT (mm) & 1.04 & 1.14 & 2,249 & 0.99 & 0.61 & 989 & $<0.001$ \\
\hline \multirow[t]{2}{*}{ Plaque score (mm) } & 2.18 & 3.10 & 2,249 & 1.10 & 2.12 & 989 & $<0.001$ \\
\hline & $n$ & $\%$ & $N$ & $n$ & $\%$ & $N$ & $\boldsymbol{P}$ \\
\hline Smoking & 60 & 4.7 & 1,273 & 0 & 0.0 & 515 & $<0.001$ \\
\hline Drinking & 68 & 5.3 & 1,205 & 1 & 0.2 & 514 & $<0.001$ \\
\hline Carotid plaque & 1,115 & 49.6 & 2,249 & 315 & 31.9 & 989 & $<0.001$ \\
\hline Plaque at CCA & 1,047 & 46.6 & 2,249 & 287 & 29.0 & 989 & $<0.001$ \\
\hline Plaque at ICA & 320 & 14.2 & 2,249 & 64 & 6.5 & 989 & $<0.001$ \\
\hline Plaque at ECA & 84 & 3.7 & 2,249 & 14 & 1.4 & 989 & $<0.001$ \\
\hline Carotid plaque & & & & & & & $<0.001$ \\
\hline \multicolumn{8}{|l|}{ composition } \\
\hline None & 1,134 & 50.4 & 2,249 & 674 & 68.1 & 989 & \\
\hline Echolucent & 440 & 19.6 & 2,249 & 125 & 12.6 & 989 & \\
\hline Echogenic & 13 & 0.6 & 2,249 & 10 & 1.0 & 989 & \\
\hline Heterogeneous & 307 & 13.7 & 2,249 & 106 & 10.7 & 989 & \\
\hline Polytype & 355 & 15.8 & 2,249 & 74 & 7.5 & 989 & \\
\hline Count of carotid plaque & & & & & & & $<0.001$ \\
\hline 0 & 1,134 & 50.0 & 2,249 & 674 & 68.1 & 989 & \\
\hline 1 & 441 & 19.6 & 2,249 & 159 & 16.1 & 989 & \\
\hline 2 & 279 & 12.4 & 2,249 & 83 & 8.4 & 989 & \\
\hline$\geq 3$ & 394 & 17.5 & 2,249 & 72 & 7.3 & 989 & \\
\hline
\end{tabular}

Plaque score is the sum of all the plaque thickness measurements. Carotid plaque composition: if all the plaques in a subject had the same composition, the subject was classified according to that composition; if the plaque had different composition, the subject was classified as having polytype plaques.

$B M I$, body mass index; SBP, systolic blood pressure; DBP, diastolic blood pressure; TC, total cholesterol; TG, triglycerides; HDLc, high-density lipoprotein cholesterol; LDLc, lowdensity lipoprotein cholesterol; WBC, white blood cell; MICPT, maximal internal carotid plaque thickness; CCA, common carotid artery; ICA, internal carotid artery; ECA, external carotid artery. 
TABLE 2 | Odds ratios and 95\% Cl for carotid artery plaque by quartiles of the total white blood cell (WBC) and differential WBC counts for men.

\begin{tabular}{|c|c|c|c|c|c|}
\hline & \multicolumn{5}{|c|}{ Odds ratios $(95 \% \mathrm{Cl})$ for carotid plaque } \\
\hline Cases, $N$ & 472 & 485 & 504 & 475 & \\
\hline Model 1 & 1.00 & $1.30(0.98-1.73)$ & $1.22(0.92-1.62)$ & $2.06(1.54-2.76)^{\star \star \star}$ & $<0.001$ \\
\hline Model 2 & 1.00 & $1.31(0.96-1.80)$ & $1.21(0.89-1.65)$ & $2.07(1.50-2.85)^{\star \star \star}$ & $<0.001$ \\
\hline \multicolumn{6}{|c|}{ Neutrophils ( $\left.\times 10^{9} / L\right)$} \\
\hline Cases, $N$ & 456 & 517 & 481 & 493 & \\
\hline Model 1 & 1.00 & $1.39(0.99-1.76)$ & $1.60(1.20-2.14)^{\star *}$ & $1.99(1.49-2.66)^{\star \star \star}$ & $<0.001$ \\
\hline Model 2 & 1.00 & $1.38(1.01-1.89)^{\star}$ & $1.76(1.28-2.42)^{\star \star \star}$ & $2.10(1.52-2.89)^{\star \star \star}$ & $<0.001$ \\
\hline Model 3 & 1.00 & $1.39(0.95-2.06)$ & $1.97(1.33-2.93)^{\star *}$ & $1.65(1.10-2.47)^{\star}$ & 0.004 \\
\hline \multicolumn{6}{|c|}{ Neutrophils (\%) } \\
\hline Model 3 & 1.00 & $1.26(0.86-1.83)$ & $1.58(1.08-2.31)^{\star}$ & $0.97(0.64-1.45)$ & 0.696 \\
\hline \multicolumn{6}{|c|}{ Lymphocytes (x 109/L) } \\
\hline Cases, $N$ & 525 & 424 & 494 & 504 & \\
\hline Model 1 & 1.00 & $1.00(0.75-1.33)$ & $1.05(0.79-1.38)$ & $1.48(1.12-1.95)^{\star \star}$ & 0.007 \\
\hline Model 2 & 1.00 & $0.91(0.66-1.25)$ & $1.05(0.77-1.42)$ & $1.36(1.00-1.85)^{\star}$ & 0.033 \\
\hline Model 3 & 1.00 & $0.88(0.59-1.32)$ & $0.98(0.65-1.40)$ & $1.41(0.96-2.08)$ & 0.076 \\
\hline \multicolumn{6}{|c|}{ Lymphocytes (\%) } \\
\hline Cases, $N$ & 473 & 500 & 468 & 480 & \\
\hline Model 1 & 1.00 & $1.06(0.80-1.40)$ & $0.94(0.71-1.25)$ & $0.84(0.63-1.12)$ & 0.155 \\
\hline Model 2 & 1.00 & $1.04(0.76-1.42)$ & $0.94(0.68-1.28)$ & $0.75(0.54-1.02)$ & 0.047 \\
\hline Cases, $N$ & 479 & 493 & 498 & 475 & \\
\hline Model 1 & 1.00 & $1.13(0.85-1.49)$ & $1.15(0.87-1.52)$ & $1.13(0.85-1.50)$ & 0.418 \\
\hline Model 2 & 1.00 & $1.10(0.81-1.49)$ & $1.17(0.86-1.59)$ & $1.14(0.84-1.56)$ & 0.367 \\
\hline Model 3 & 1.00 & $1.07(0.73-1.57)$ & $1.18(0.80-1.76)$ & $1.28(0.87-1.89)$ & 0.179 \\
\hline \multicolumn{6}{|c|}{ Eosinophils ( $\times 10^{9} /$ L) } \\
\hline Cases, $N$ & 531 & 448 & 470 & 497 & \\
\hline Model 1 & 1.00 & $1.23(0.96-1.62)$ & $1.29(0.97-1.70)$ & $1.31(1.00-1.73)$ & 0.049 \\
\hline Model 2 & 1.00 & $1.29(0.94-1.75)$ & $1.27(0.93-1.73)$ & $1.35(0.99-1.82)$ & 0.071 \\
\hline Model 3 & 1.00 & $1.51(1.02-2.23)^{\star}$ & $1.55(1.05-2.28)^{\star}$ & $1.28(0.88-1.88)$ & 0.200 \\
\hline \multicolumn{6}{|c|}{ Eosinophils (\%) } \\
\hline Cases, $N$ & 475 & 479 & 504 & 489 & \\
\hline Model 1 & 1.00 & $0.81(0.61-1.08)$ & $1.00(0.78-1.32)$ & $0.89(0.67-1.18)$ & 0.767 \\
\hline Model 2 & 1.00 & $0.81(0.60-1.10)$ & $0.95(0.70-1.28)$ & $0.86(0.63-1.17)$ & 0.543 \\
\hline Model 3 & 1.00 & $0.92(0.62-1.35)$ & $1.33(0.91-1.95)$ & $1.07(0.73-1.57)$ & 0.359 \\
\hline \multicolumn{6}{|c|}{ Basophils (x $\left.10^{9} / \mathrm{L}\right)$} \\
\hline Cases, $N$ & 556 & 566 & 389 & 434 & \\
\hline Model 1 & 1.00 & $1.17(0.90-1.53)$ & $1.35(1.01-1.81)^{\star}$ & $1.42(1.07-1.89)^{\star}$ & 0.009 \\
\hline Model 2 & 1.00 & $1.16(0.88-1.54)$ & $1.45(1.05-1.99)^{\star}$ & $1.46(1.07-2.00)^{\star}$ & 0.007 \\
\hline Model 3 & 1.00 & $1.01(0.70-1.44)$ & $1.21(0.81-1.82)$ & $1.45(0.99-2.13)$ & 0.039 \\
\hline
\end{tabular}




\begin{tabular}{|c|c|c|c|c|c|}
\hline & \multicolumn{5}{|c|}{ Odds ratios $(95 \% \mathrm{Cl})$ for carotid plaque } \\
\hline \multicolumn{6}{|c|}{ Basophils (\%) } \\
\hline Cases, $N$ & 333 & 470 & 752 & 392 & \\
\hline Model 1 & 1.00 & $0.91(0.66-1.24)$ & $1.01(0.75-1.34)$ & $0.99(0.71-1.37)$ & 0.806 \\
\hline Model 2 & 1.00 & $0.88(0.62-1.24)$ & $1.01(0.73-1.38)$ & $1.06(0.74-1.51)$ & 0.515 \\
\hline
\end{tabular}

Model 1 was adjusted for age and BMI.

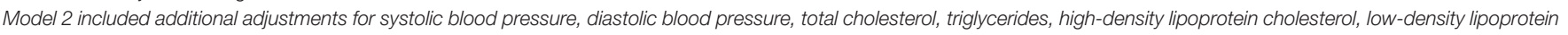
cholesterol, fasting plasma glucose, and uric acid.

Model 3 included additional adjustments for drinking and smoking.

${ }^{\star} P \leq 0.05,{ }^{\star \star} P \leq 0.01$, and ${ }^{\star \star \star} P \leq 0.001$ compared with $Q 1$ (Bonferroni). Bold $P$ indicates statistical significance.

\section{WBC and the Presence of CAPs}

In men, the total WBC, neutrophil, and monocyte counts showed significant positive correlations with the presence of CAPs after adjustment for age, BMI, SBP, DBP, TC, TG, HDLc, LDLc, fasting glucose, and uric acid $\left(P<0.001, P_{\text {trend }}<0.001\right.$; Table 2). In women, the total WBC and differential WBC counts showed no significant association with the presence of CAPs (Table 3). The associations of the total WBC, neutrophil, and monocyte counts with the presence of CAPs existed even after further adjustment for smoking and drinking. The odds ratios (95\% CI) of the individuals with CAP in the highest (compared to the lowest) quartile were 1.99 (1.33-2.97), 1.65 (1.10-2.47), and 2.17 $(1.41-3.18)\left(P_{\text {trend }}=0.004, P_{\text {trend }}=0.004\right.$, and $P_{\text {trend }}<0.001$, respectively) for the total $\mathrm{WBC}$, neutrophil, and monocytes count, respectively. Similar, but less robust, results were found for the percentages of neutrophils, lymphocytes, and basophils $(P<$ $\left.0.05, P_{\text {trend }}<0.05\right)$. Further analysis revealed that the significant associations of the total WBC, neutrophil, and monocyte counts with the presence of CAPs only existed in men aged 45-65 years (Tables 4, 5). Additionally, restrictive cubic spline showed that the total WBC, neutrophil, and monocyte counts had a nonlinear relationship with the presence of CAPs, consistent with the main results (Figures S2-S4).

\section{WBCs and the Severity of CAPs}

In the total study population, the total WBC, neutrophil, and monocyte counts were significantly correlated with the severity of carotid atherosclerosis, as judged by either the sum of CAPs, MICPT, or the plaque score (all $P<0.01, P_{\text {trend }}<0.05$; Tables S1-S3). Similar results were found for the percentage of lymphocytes $(P<0.05)$.

\section{WBCs and the Types of CAPs}

Significant differences were observed between participants without CAPs and participants with different subtypes of CAPs (Table S4). Compared with the participants without CAPs, those with echolucent plaques showed significantly increased total WBC and neutrophil counts, but significantly decreased percentage of lymphocytes, whereas those with polytype plaques showed significantly increased monocyte counts.

\section{DISCUSSION}

To our knowledge, the current study is the first to investigate the associations of the total WBC and WBC subtype counts with the presence, severity, and subtypes of CAPs in a healthy Chinese population. Our results suggest that the total WBC and WBC subtype counts contain important risk information for CAPs. The total WBC, neutrophil, and monocyte counts were significantly associated with increased odds of the presence of CAPs in men, but not in women after adjustment for potential confounding factors. These three counts also showed significant positive associations with the severity and subtypes of CAPs.

Several epidemiologic observational studies have reported that an increased WBC count is associated with a higher cardiovascular risk prediction $(8,9)$. The total WBC and neutrophil counts are also confirmed as risk factors for the outcomes of myocardial infarction and cerebral infarction $(16,17)$ and are associated with infarction size $(18)$. Interestingly, the neutrophil count has been found to be significantly associated with the angiographic characteristics of coronary artery disease, strongly suggesting that neutrophil was involved in the development of coronary atherosclerosis (19). The results of our study not only confirm the positive relationship between the total WBC count and CAPs but also show that neutrophils and monocytes are superior to other WBC subtypes in indicating the presence of CAPs.

Gender differences in the prevalence and complications of atherosclerosis are well-known. However, data are limited in the clinical and preclinical literature to provide robust evidence regarding the underlying mechanisms. Genetic background and biological heterogeneity might play essential roles in the gender difference observed in atherosclerosis (20). Moreover, the influence of sex hormones on atherosclerosis is still controversial (21). Research indicates that although younger women have a lower risk of cardiovascular disease than men, women's risk catches up to and even surpasses that of men at the age of 60-79 years (22). Moreover, the risk factors for atherosclerosis might differ between genders. In our study, the overall BMI, blood pressure, and lipid profiles were 
TABLE 3 | Odds ratios and 95\% Cl for carotid artery plaque by quartiles of total white blood cell (WBC) and differential WBC counts for women.

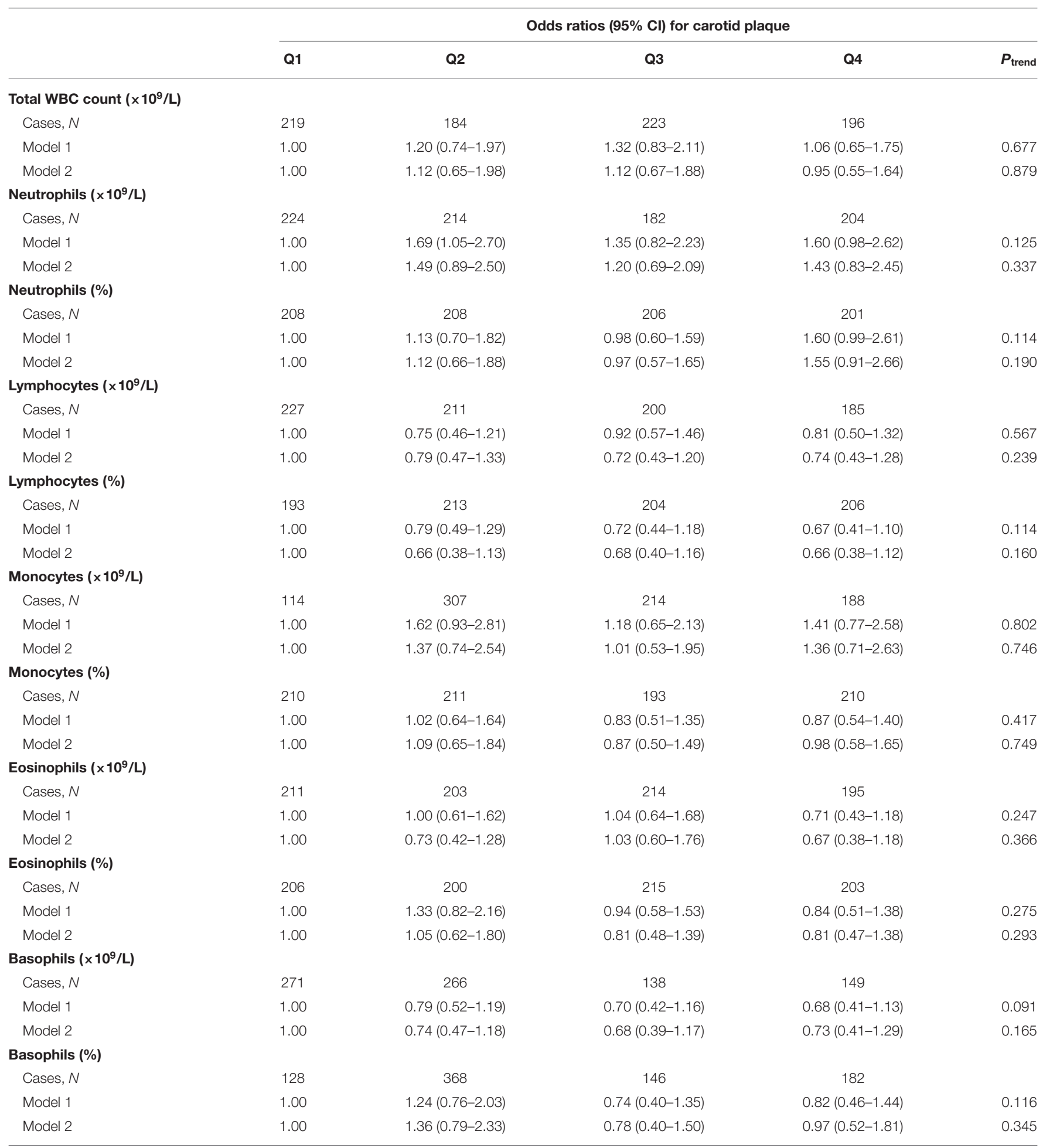

Model 1 was adjusted for age and BMI.

Model 2 included additional adjustments for systolic blood pressure, diastolic blood pressure, total cholesterol, triglycerides, high-density lipoprotein cholesterol, low-density lipoprotein cholesterol, fasting plasma glucose, and uric acid.

significantly lower in women, which might have contributed to the differential results in the association between leukocytes and CAPs.
Studies have shown that CAP burden might be an independent indicator of the severity of intracranial artery atherosclerosis and can also predict future cardiovascular and 
TABLE 4 | Adjusted odds ratios and 95\% Cl for carotid artery plaque stratified by age for men.

\begin{tabular}{|c|c|c|c|c|c|}
\hline & \multicolumn{5}{|c|}{ Adjusted odds ratios $(95 \% \mathrm{Cl})$ for carotid plaque } \\
\hline$<45$ years & 1.00 & $1.50(0.64-3.53)$ & $1.64(0.72-3.70)$ & $1.85(0.83-4.10)$ & 0.170 \\
\hline $45-65$ years & 1.00 & $1.16(0.81-1.65)$ & $1.11(0.78-1.58)$ & $1.82(1.28-2.59)^{\star \star \star}$ & $<0.001$ \\
\hline$\geq 65$ years & 1.00 & $1.80(0.46-7.09)$ & $0.70(0.18-2.71)$ & $1.74(0.44-6.92)$ & 0.479 \\
\hline \multicolumn{6}{|c|}{ Neutrophils (×109/L) } \\
\hline$<45$ years & 1.00 & $2.44(0.96-6.20)$ & $2.96(1.17-7.48)^{\star}$ & $3.18(1.26-8.03)^{\star}$ & 0.071 \\
\hline $45-65$ years & 1.00 & $1.26(0.88-1.81)$ & $1.59(1.12-2.25)^{\star \star}$ & $1.93(1.35-2.78)^{\star \star \star}$ & 0.012 \\
\hline$\geq 65$ years & 1.00 & $1.32(0.35-5.02)$ & $3.46(0.73-16.41)$ & $1.55(0.41-5.93)$ & 0.218 \\
\hline$P_{\text {interaction }}$ & 0.333 & & & & \\
\hline \multicolumn{6}{|c|}{ Lymphocytes (x 109/L) } \\
\hline$P_{\text {interaction }}$ & 0.426 & & & & \\
\hline \multicolumn{6}{|c|}{ Monocytes (×109/L) } \\
\hline$<45$ years & 1.00 & $1.60(0.76-3.36)$ & $0.87(0.41-1.85)$ & $1.31(0.62-2.77)$ & 0.770 \\
\hline $45-65$ years & 1.00 & $1.15(0.82-1.62)$ & $1.56(1.09-2.23)^{\star}$ & $2.29(1.59-3.30)^{\star \star \star}$ & $<0.001$ \\
\hline$\geq 65$ years & 1.00 & $3.29(0.78-13.78)$ & $0.99(0.28-3.49)$ & $24.40(2.48-239.75)^{\star \star}$ & 0.104 \\
\hline$P_{\text {interaction }}$ & 0.341 & & & & \\
\hline \multicolumn{6}{|c|}{ Eosinophils ( $\times 10^{9} /$ L) } \\
\hline$<45$ years & 1.00 & $1.63(0.75-3.52)$ & $1.70(0.80-3.60)$ & $1.28(0.58-2.81)$ & 0.602 \\
\hline $45-65$ years & 1.00 & $1.06(0.73-1.53)$ & $1.16(0.82-1.64)$ & $1.23(0.87-1.75)$ & 0.584 \\
\hline$\geq 65$ years & 1.00 & $2.53(0.66-9.67)$ & $2.41(0.68-8.51)$ & $7.13(1.61-31.64)$ & 0.394 \\
\hline
\end{tabular}

Model adjusted for BMI, systolic blood pressure, diastolic blood pressure, total cholesterol, triglycerides, high-density lipoprotein cholesterol, low-density lipoprotein cholesterol, fasting plasma glucose, and uric acid.

WBC, white blood cell.

${ }^{*} P \leq 0.05,{ }^{* *} P \leq 0.01$, and ${ }^{* \star} P \leq 0.001$ compared with Q1 (Bonferroni). Bold P indicates statistical significance.

cerebrovascular events (23). Hence, CAP burden may be more meaningful than just the presence or absence of a carotid plaque. A study among Hispanics found that individuals in the highest quartile of the WBC count had significantly increased MICPT compared with the subjects in the lowest quartile (13). Another study in non-smoking men $(n=571)$ found that the presence and severity of carotid atherosclerosis were significantly associated with increased counts of total WBCs, neutrophils, and monocytes (24). These results are consistent with our finding that the total WBC, neutrophil, and monocyte counts were significantly positively correlated with the sum of CAPs, MICPT, and the plaque score.

The risk of rupture of atherosclerotic plaques is a main concern in carotid atherosclerosis. Generally, the stability of CAP could be indicated by its compositions. Echolucent or hypoechogenic plaques were mainly composed of lipids, inflammatory cells, and neovessels. However, echogenic plaques mainly comprise calcified tissues (25). Previous studies have found that compared to echogenic plaques, echolucent plaques are associated with cerebrovascular events and future coronary events (26). The leukocyte count has been reported to be associated with the degree of CAP instability (27), and a high neutrophil count in CAPs is related to the features of ruptureprone lesions (28). In our study, we also found that, compared with the participants without CAP, those with echolucent plaques showed significantly increased total WBC and neutrophils counts, whereas those with polytype plaques showed significantly increased monocyte counts.

Several pathophysiological mechanisms potentially link the WBCs and WBC subtypes to plaque formation and vulnerability. First of all, recruitment of leukocytes to the vessel endothelium wall represents an early stage in the development 
TABLE 5 | Adjusted odds ratios and 95\% Cl for carotid artery plaque stratified by age for women.

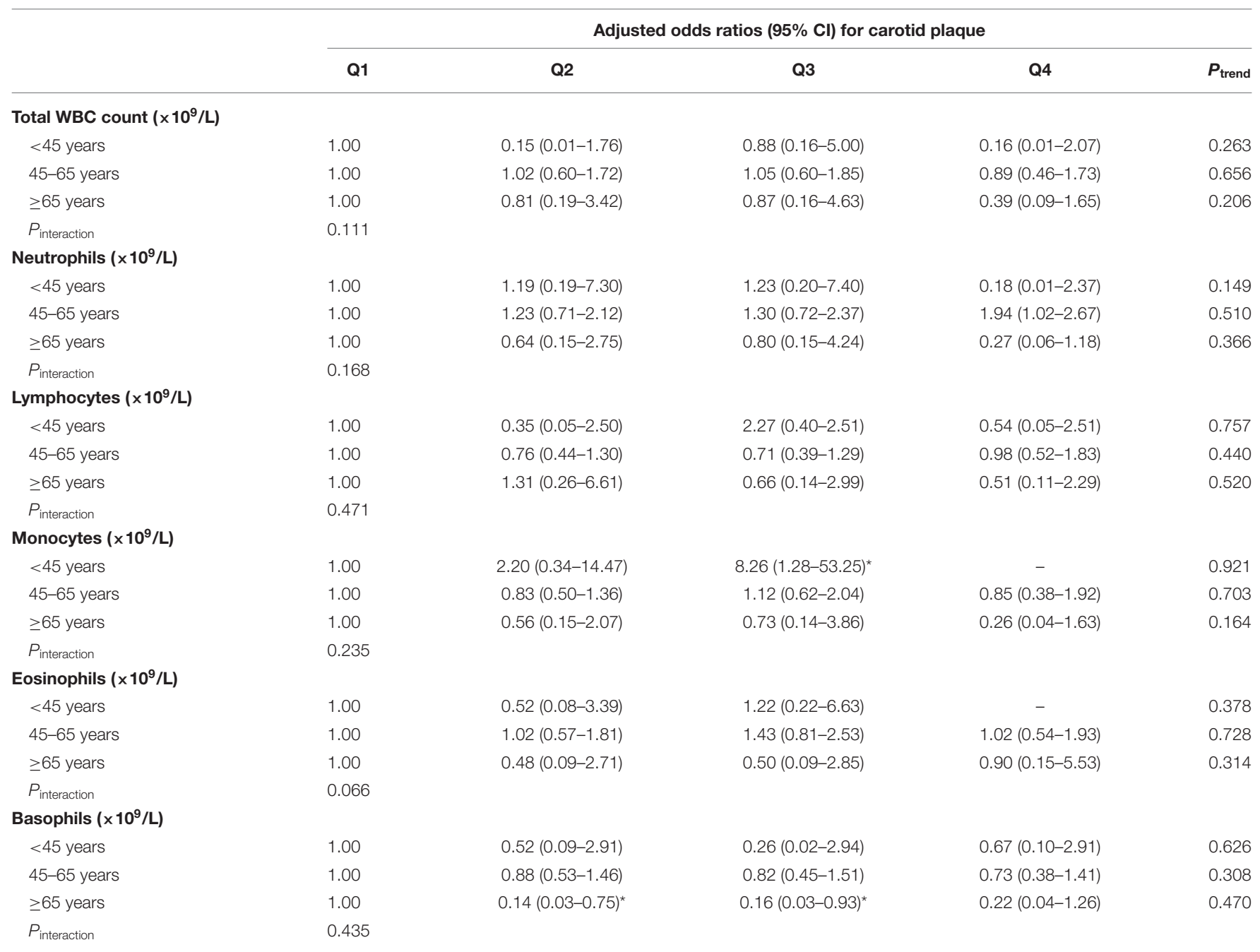

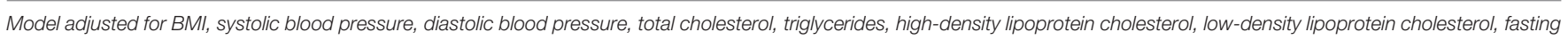
plasma glucose, and uric acid.

WBC, white blood cell.

${ }^{*} P \leq 0.05$ compared with Q1 (Bonferroni).

of atherosclerosis. CAP is characterized by the infiltration of inflammatory cells, which transmigrated from blood into the subendothelial layers of the arteries (29). Monocytes are the primary inflammatory infiltrating cells in early atherosclerotic plaques, and their accumulation increased proportionally with plaque size (30). The presence of neutrophil invasion in atherosclerotic plaques has been shown in an animal model and in patients with acute coronary syndrome (31). Neutrophils localized in rupture-prone areas and released active mediators, which caused plaque instability (28). Secondly, inflammation cells play a vital role in the progress of atherosclerosis and plaque vulnerability (32). Monocytes, as representatives of the innate immune system, could drive the progression of atherosclerosis from a stable to an unstable state $(33,34)$. Neutrophils also promoted the progression of atherosclerosis because of their ability to activate other immune cells, especially monocytes, instructing them to directly participate in the inflammation, and/or release pro-inflammatory mediators. Furthermore, neutrophils might increase plaque vulnerability by releasing various enzymes, including gelatinase, collagenase, myeloperoxidase, and elastase (35).

This study has some limitations to acknowledge. Firstly, it was designed as a cross-sectional observational study, so no causal relationship can be drawn. Our results should be further investigated in a large prospective cohort. Secondly, the crosssectional design may lead to a selection bias, especially with respect to the healthy elderly. However, excluding subjects with histories of cardiovascular diseases might partially decrease the bias. Thirdly, the study population was less likely to be smokers than the general Chinese population. Therefore, caution should be exercised when generalizing the results. Finally, the assessment of CAP with ultrasound may be less reliable than with magnetic resonance imaging or computed tomography. However, considering its convenience and the likelihood of 
technical improvements, ultrasound still remains the first choice for CAP assessment.

In conclusion, this study found that the total WBC, neutrophil, and monocyte counts are associated with the presence, severity, and types of CAPs in healthy Chinese adults, and these markers may be useful in clinical practice.

\section{DATA AVAILABILITY STATEMENT}

The datasets generated for this study are available on request to the corresponding author.

\section{ETHICS STATEMENT}

The studies involving human participants were reviewed and approved by Research Ethical Committee of the First Affiliated Hospital of Zhengzhou University. The patients/participants provided their written informed consent to participate in this study.

\section{REFERENCES}

1. GBD 2013 Mortality and Causes of Death Collaborators. Global, regional, and national age-sex specific all-cause and cause-specific mortality for 240 causes of death, 1990-2013: a systematic analysis for the Global Burden of Disease Study 2013. Lancet. (2015) 385:117-71. doi: 10.1016/S0140-6736(14)61682-2

2. Gao F, Sun RJ, Ji Y, Yang BF. Cardiovascular research is thriving in China. Br J Pharmacol. (2015) 172:5430-4. doi: 10.1111/bph.12826

3. Sturlaugsdottir R, Aspelund T, Bjornsdottir G, Sigurdsson S, Thorsson B, Eiriksdottir G, et al. Prevalence and determinants of carotid plaque in the cross-sectional REFINE-Reykjavik study. BMJ open. (2016) 6:e012457. doi: 10.1136/bmjopen-2016-012457

4. Grossmann V, Schmitt VH, Zeller T, Panova-Noeva M, Schulz A, LaubertReh D, et al. Profile of the immune and inflammatory response in individuals with prediabetes and type 2 diabetes. Diabetes Care. (2015) 38:1356-64. doi: $10.2337 / \mathrm{dc} 14-3008$

5. Nunez J, Sastre C, D’Ascoli G, Ruiz V, Bonanad C, Minana G, et al. Relation of low lymphocyte count to frailty and its usefulness as a prognostic biomarker in patients $>65$ years of age with acute coronary syndrome. Am J Cardiol. (2020) 125:1033-8. doi: 10.1016/j.amjcard.2020.01.006

6. Siedlinski M, Jozefczuk E, Xu X, Teumer A, Evangelou E, Schnabel RB, et al. White blood cells and blood pressure: a Mendelian Randomization study. Circulation. (2020) 141:1307-17. doi: 10.1161/CIRCULATIONAHA.119.045102

7. Macrez R, Ali C, Toutirais O, Le Mauff B, Defer G, Dirnagl U, et al. Stroke and the immune system: from pathophysiology to new therapeutic strategies. Lancet Neurol. (2011) 10:471-80. doi: 10.1016/S1474-4422(11)70066-7

8. Furlan JC, Vergouwen MD, Fang J, Silver FL. White blood cell count is an independent predictor of outcomes after acute ischaemic stroke. Eur J Neurol. (2014) 21:215-22. doi: 10.1111/ene.12233

9. Taglieri N, Bacchi Reggiani ML, Palmerini T, Cinti L, Saia F, Guastaroba P, et al. Baseline white blood cell count is an independent predictor of long-term cardiovascular mortality in patients with non-ST-segment elevation acute coronary syndrome, but it does not improve the risk classification of the GRACE score. Cardiology. (2013) 124:97-104. doi: 10.1159/000346377

10. Swirski FK, Nahrendorf M. Leukocyte behavior in atherosclerosis, myocardial infarction, and heart failure. Science. (2013) 339:161-6. doi: $10.1126 /$ science. 1230719

11. Sarlon-Bartoli G, Bennis Y, Lacroix R, Piercecchi-Marti MD, Bartoli MA, Arnaud L, et al. Plasmatic level of leukocyte-derived microparticles is associated with unstable plaque in asymptomatic patients with

\section{AUTHOR CONTRIBUTIONS}

YX and YL were the project lead for the current study. YZ, WJ, DS, and LZ established the database and extracted data. YL, YC, and $\mathrm{CZ}$ conducted data and statistical analysis. YL, YZ, and GC wrote the manuscript. QL and YC provided significant advice on the manuscript. YB and YX reviewed and revised the manuscript. All authors read and approved the final manuscript.

\section{FUNDING}

This present study was supported by the National Natural Science Foundation of China (81602852).

\section{SUPPLEMENTARY MATERIAL}

The Supplementary Material for this article can be found online at: https://www.frontiersin.org/articles/10.3389/fmed. 2020.00313/full\#supplementary-material

high-grade carotid stenosis. J Am Coll Cardiol. (2013) 62:1436-41. doi: 10.1016/j.jacc.2013.03.078

12. Stein JH KC, Hurst RT, Lonn E, Kendall CB, Mohler ER, Najjar SS, et al. Use of carotid ultrasound to identify subclinical vascular disease and evaluate cardiovascular diseaserisk: a consensus statement from the American society of echocardiography carotid intima-mediathickness task force. Endorsed by the society for vascular medicine. J Am Soc Echocardiogr. (2008) 21:93-111. doi: 10.1016/j.echo.2007.11.011

13. Elkind MS, Cheng J, Boden-Albala B, Paik MC, Sacco RL. Elevated white blood cell count and carotid plaque thickness: the northern Manhattan Stroke study. Stroke. (2001) 32:842-9. doi: 10.1161/01.STR.32.4.842

14. Kawai T, Ohishi M, Takeya Y, Onishi M, Ito N, Oguro R, et al. Carotid plaque score and intima media thickness as predictors of stroke and mortality in hypertensive patients. Hypertens Res. (2013) 36:902-9. doi: 10.1038/hr.2013.61

15. Vigili de Kreutzenberg S, Fadini GP, Guzzinati S, Mazzucato M, Volpi A, Coracina A, et al. Carotid plaque calcification predicts future cardiovascular events in type 2 diabetes. Diabetes Care. (2015) 38:1937-44. doi: $10.2337 / \mathrm{dc} 15-0327$

16. Palmerini T, Brener SJ, Genereux P, Maehara A, Della Riva D, Mariani A, et al. Relation between white blood cell count and final infarct size in patients with ST-segment elevation acute myocardial infarction undergoing primary percutaneous coronary intervention (from the INFUSE AMI trial). Am J Cardiol. (2013) 112:1860-6. doi: 10.1016/j.amjcard.2013.08.010

17. Peng Y, Wang D, Zhang J, Xue X, Wang Z, Tong W, et al. Relationship between white blood cell count at admission and short term outcome in patients with acute cerebral infarction. Clin Invest Med. (2011) 34:E249. doi: $10.25011 /$ cim.v34i4.15368

18. Husser O, Bodi V, Sanchis J, Nunez J, Mainar L, Chorro FJ, et al. White blood cell subtypes after STEMI: temporal evolution, association with cardiovascular magnetic resonance-derived infarct size and impact on outcome. Inflammation. (2011) 34:73-84. doi: 10.1007/s10753-010-9209-0

19. Jia EZ, Yang ZJ, Yuan B, Zang XL, Wang RH, Zhu TB, et al. Relationship between leukocyte count and angiographical characteristics of coronary atherosclerosis. Acta Pharmacol Sin. (2005) 26:1057-62. doi: 10.1111/j.1745-7254.2005.00169.x

20. Man JJ, Beckman JA, Jaffe IZ. Sex as a biological variable in atherosclerosis. Circ Res. (2020) 126:1297-319. doi: 10.1161/CIRCRESAHA.120.315930

21. Morselli E, Santos RS, Criollo A, Nelson MD, Palmer BF, Clegg DJ. The effects of oestrogens and their receptors on cardiometabolic health. Nat Rev Endocrinol. (2017) 13:352-64. doi: 10.1038/nrendo.2017.12 
22. Virani SS, Alonso A, Benjamin EJ, Bittencourt MS, Callaway CW, Carson AP, et al. Heart disease and stroke statistics-2020 update: a report from the American Heart Association. Circulation. (2020) 141:e139-e596. doi: 10.1161/CIR.0000000000000746

23. Xu Y, Li D, Yuan C, Zhou Z, He L, Li R, et al. Association of severity between carotid and intracranial artery atherosclerosis. Ann Clin Transl Neurol. (2018) 5:843-9. doi: 10.1002/acn3.590

24. Huang ZS, Jeng JS, Wang CH, Yip PK, Wu TH, Lee TK. Correlations between peripheral differential leukocyte counts and carotid atherosclerosis in non-smokers. Atherosclerosis. (2001) 158:431-6. doi: 10.1016/S0021-9150(01)00445-2

25. Lal BK, Hobson RW 2nd, Pappas PJ, Kubicka R, Hameed M, Chakhtoura EY, et al. Pixel distribution analysis of B-mode ultrasound scan images predicts histologic features of atherosclerotic carotid plaques. J Vasc Surg. (2002) 35:1210-7. doi: 10.1067/mva.2002.122888

26. Aldemir E, Apaydin M, Varer M, Uluc E. Echolucency of carotid plaques and cerebrovascular events. J Clin Ultrasound. (2012) 40:399-404. doi: 10.1002/jcu.21951

27. Minic GA. Leucocyte count indicates carotid plaque instability in stroke patients. Vojnosanit Pregl. (2016) 73:515-25. doi: 10.2298/VSP141222131A

28. Ionita MG, van den Borne P, Catanzariti LM, Moll FL, de Vries JP, Pasterkamp $G$, et al. High neutrophil numbers in human carotid atherosclerotic plaques are associated with characteristics of rupture-prone lesions. Arterioscler Thromb Vasc Biol. (2010) 30:1842-8. doi: 10.1161/ATVBAHA.110.209296

29. Shah PK, Galis ZS. Matrix metalloproteinase hypothesis of plaque rupture: players keep piling up but questions remain. Circulation. (2001) 104:1878-80. doi: 10.1161/circ.104.16.1878

30. Weber C, Zernecke A, Libby P. The multifaceted contributions of leukocyte subsets to atherosclerosis: lessons from mouse models. Nat Rev Immunol. (2008) 8:802-15. doi: 10.1038/nr i 2415

31. Naruko T, Ueda M, Haze K, van der Wal AC, van der Loos CM, Itoh A, et al. Neutrophil infiltration of culprit lesions in acute coronary syndromes. Circulation. (2002) 106:2894-900. doi: 10.1161/01.CIR.0000042674.89762.20

32. Schiopu A, Cotoi OS. S100A8 and S100A9: DAMPs at the crossroads between innate immunity, traditional risk factors, and cardiovascular disease. Mediators Inflamm. (2013) 2013:828354. doi: 10.1155/2013/828354

33. Ghattas A, Griffiths HR, Devitt A, Lip GY, Shantsila E. Monocytes in coronary artery disease and atherosclerosis: where are we now? J Am Coll Cardiol. (2013) 62:1541-51. doi: 10.1016/j.jacc.2013.07.043

34. Randolph GJ. The fate of monocytes in atherosclerosis. J Thromb Haemost. (2009) 7(Suppl. 1):28-30. doi: 10.1111/j.1538-7836.2009.0 3423.x

35. Carbone F, Mach F, Montecucco F. Update on the role of neutrophils in atherosclerotic plaque vulnerability. Curr Drug Targets. (2015) 16:321-33. doi: $10.2174 / 1389450115666141110093013$

Conflict of Interest: The authors declare that the research was conducted in the absence of any commercial or financial relationships that could be construed as a potential conflict of interest.

Copyright (c) 2020 Liu, Zhu, Jia, Sun, Zhao, Zhang, Wang, Lyu, Chen, Chen, Bo and Xing. This is an open-access article distributed under the terms of the Creative Commons Attribution License (CC BY). The use, distribution or reproduction in other forums is permitted, provided the original author(s) and the copyright owner(s) are credited and that the original publication in this journal is cited, in accordance with accepted academic practice. No use, distribution or reproduction is permitted which does not comply with these terms. 